\title{
Un exercice de rédaction: la contraction de texte
}

\author{
I.ionel Mintey \\ Université Lanal
}

\section{Introduction}

LA NÉCESSITÉ D'AMÉLIORER LES QUALITÉS rédactionnelles des étudiants se fait sentir depuis plusieurs années dans toutes les sociétés occidentales. Dans les progrạmmes de rédaction technique, de traduction ou de journalisme, la contraction de texte s'est révélée un moyen très efficace pour atteindre cet objectif. La présentation qui suit est le résultat de l'expérience de plusieurs années d'enseignement de cet exercice en première année d'université en contexte francophone canadien. Certains de nos choix suivent des usages que l'on peut observer dans la plupart des manuels de contraction de texte; ${ }^{1}$ d'autres sont influencés par les études théoriques qui ont été publiées au cours des deux dernières decomies; ${ }^{2}$ d'autres enfin sont le fruit de nos observations et de notre expérience dans le cadre évoqué cidessus.

\section{Principes generaux}

\subsection{Définition}

La contraction de texte est un exercice de rédaction qui consiste à réduire un texte donné (texte de départ ou TD) dans des proportions déterminées à l'avance, tout en conservant dans le texte d'arrivée (ou TA) l'essentiel du message de TD, selon la formule: Message de TA = Essentiel du message de TD.

1 Voir Bibliographie: Manuels pratiques. La plupart des manuels de contraction de texte ne donnent que des indications méthodologiques très générales. Il semble que la recherche théorique n'ait pas encore été intégré par leurs auteurs.

2 Voir Bibliograplie: Etudes théoriques. 
La langue de seut être la même que celle de TD ou différente. Nous

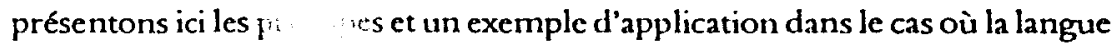
de TA est la même. . He de TD, le français. les antres cas de figure (par

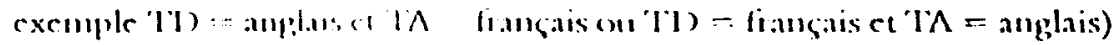
posent dianes publitures que nous ne pouvons aborder dans le cadre de irtir ćluik.

Même si les auteurs ne sont pas toujours d'accord sur ce point, ${ }^{3}$ il convient, selon nous, de distinguer résumé et contraction. Ce dernier mot implique en effet la notion de limites précises à respecter et de réduction proportionnelle du texte de départ. La notion de résumé nous semble à cet égard moins contraignante. Or, une part essentielle de l'intérêt pédagogique de l'exercice dépend de ces deux règles, certes arbitraires mais irremplaçables dans leur exigence, de respect de limites contraignantes et de proportions relatives de réduction à observer entre les différentes composantes du texte.

Par ailleurs, il est important d'insister sur la nécessité de rendre, d'une manière impartiale et sans intervention perceptible de l'étudiant, le message de l'auteur. A cet égard, la contraction se différencie de l'analyse de texte qui, au contraire, exige la présence et le jugement de l'étudiant.

\subsection{Règles du jeu}

Ces règles ont, comme nous venons de le dire, un caractère assez arbitraire, mais elles sont nécessaires. Bien entendu, celles que nous proposons ici n'ont rien d'absolu et peuvent être modifiées en fonction des objectifs pédagogiques. Les voici résumées sous forme de consignes:

Règle n" 1: Réduise: le texte proposé dans des proportions déterminées (voir ci-dessous).

Règle $n^{\circ} 2$ : Retenez les informations les plus importantes.

Règle $n^{\circ}$ 3: Respectez le message de l'auteur.

Règle $n^{\circ} 4$ : Respectez l'ordre d'apparition des idées dans le texte.

Règle $n^{\circ}$ 5: Rédigez un texte qui soit compréhensible en lui-même.

Règle $n^{\circ} 6$ : Ne copiez pas le style de l'auteur, reformulez ses idées dans un style simple et neutre.

Règle $n^{\circ} 7$ : Ne manifestez pas votre présence dans votre texte.

3 Les deux termes sont employés. Au baccalauréat français, on parle de résumé; aux concours des grandes ćcoles et dans l'enøeignement supéricur, on parle plutôt de contraction de textc. 
Autres données:

- Longueur des textes (TD): Elle peut varier de quelques centaines de mots à quelques milliers.

- Taille de la contraction (TA): Elle peut être exprimée sous forme de fraction (Réduisez au $1 / 4$, au $1 / 3$, au $1 / 5$, au $1 / 10$ le texte proposé) ou en nombre de mots (Résumez ou Contractez en 150 mots, en 300 mots, etc.).

- Tolérance: Une tolérance de plus ou moins 5 ou 10 p. cent dans le décompte des mots est accordée.

- Décompte des mots: I'unite choisic est la définition typographicjue:

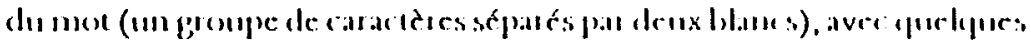
accommodements pour l'apostrophe, le trait d'union, cer.

- Titre: Le titre de la contraction doit être de type informatif. Il fait partie du décompte des mots.

\subsection{Avantages de la contraction de texte}

Il est très important d'expliquer aux étudiants la raison du choix de cet exercice, car bien souvent cela ne leur semble pas très clair, surtout au début.

Il convient d'abord de souligner que c'est un exercice qui permet de développer l'aptitude à la compréhension des textes; qu'il favorise le développement des deux démarches fondamentales de tout travail intellectuel, l'analyse et la synthèse.

Il faut dire aussi que de nombreux textes de la vie courante ou professionnelle sont proches de la contraction ou impliquent les mêmes opérations de rédaction. C'est vrai pour les présentations de films dans les programmes de cinéma, les abstracts des publications scientifiques, les articles de journaux ou de revues faisant le compte rendu d'un rapport ou d'une étude, les comptes rendus de réunion, etc. Malgré les apparences, il ne s'agit donc absolument pas d'un exercice détaché de la réalité, même de la réalité professionnelle.

Les avantages pédagogiques sont certainement très importants. La contraction de texte est un exercice très économique, et pour le professeur et pour l'étudiant, et qui permet d'aborder la plupart des problèmes de rédaction. Economique et pratique pour le professeur, parce qu'elle est de longueur modeste (donc rapidité de correction), que chaque étudiant travaille sur le même texte (donc facilité de comparaison), que la correction en classe sera mieux suivie par tous (puisqu on parle de la même chose). Economique pour l'étudiant parce que, contrairement à la dissertation ou à l'essai, celui-ci n'a 
pas besoin de faire de longues recherches en bibliothèque: initié à la méthode, il peut travailler, chez lui ou en classe, à l'aide de seulement quelques ouvrages (par ex. les Petit Robert 1 et 2).

Or, la contraction permet de travailler plusieurs aspects de la rédaction: l'expression exacte et correcte des idées, les transitions, la cohérence textuelle, la propriété des termes, la correction orthographique et grammaticale, la ponctuation, la présentation, etc.

\subsection{Les étapes de la contraction}

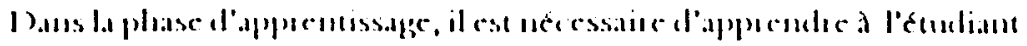
a suivic pas a pass les chapes suivantes:

a) Lecture globale du texte de départ

Lobjectif est de prendre connaissance du texte, d'en déterminer le thème et, s'il y a lieu, le message essentiel. Il est utile à cette étape de souligner les mots et les expressions, les noms propres et les allusions qui font difficulté et demanderont une recherche ultérieure. Il convient de mettre l'accent sur les conditions de production du texte et la situation dénonciation afin, entre autres, d'éviter des erreurs d'interprétation.

b) Recherche lexicale et documentaire

L'objectif est de vérifier, s'il y a lieu, dans un bon dictionnaire de langue et une encyclopédie les allusions et les mots repérés lors de la première lecture. C'est l'occasion pour le professeur $d$ 'insister sur l'importance de la compréhension du texte et sur la nécessité pour y parvenir de comprendre parfaitement le vocabulaire, les allusions, les conditions de production, la situation d'énonciation.

c) Lecture analytique du texte de départ

- Détermination du mouvement du texte

Lobjectif est de saisir le mouvement du texte, le cheminement de la pensée de l'auteur. Il convient en particulier de repérer, d'entourer et d'analyser les connecteurs. Il y a lieu aussi de repérer les passages où les transitions sont implicites. Cette étape est d'une importance fondamentale, car, dans la mesure où l'étudiant aura bien saisi le mouvement du texte, il y a de bonnes chances pour que sa contraction en rende compte correctement. 
- Choix des idées à retenir

C'est l'étape la plus difficile à franchir. Celle qui justifie l'exercice. Celle qui est la moins expliquée dans les ouvrages spécialisés. Elle doit s'appuyer sur lc jugement, en partant du principe que chacun est apte à juger de l'importance relative des idécs d'un texte et qu'il est possible, pour un groupe de personnes, de tomber d'accord sur le choix de ces idées ${ }^{4}$

d) Rédaction du plan

Le plan doit être conçu comme le moyen de passage, de transfert, du texte de départ au texte d'arrivée. L'objectif est de conserver l'organisation originelle et de mettre en valeur leschamières. Il sera rédigé de préférence sous forme nominale: La nominalisation est un excellent moyen de réexpression des idées qui permet d'éviter le défaut de l'imitation stylistique de l'original.

e) Rédaction d'une première version

Le plan étant établi, la première rédaction de la contraction devrait se faire à partir de lui seul, sans retour au texte de départ, afin d'éviter, une fois de plus, le danger d'imitation du texte de l'auteur. Cette première version a pour objectif de mettre en place les idées selon leur organisation et leur importance relative. A cette étape, l'étudiant ne devrait pas s'attarder à la correction de la langue.

D Comparaison du texte d'arrivec avec le texte de départ

Lobjectifest de vénficr que toutes les idlées impuntantes sont présentes dans la contraction, qu elles sont exprimés avec exactitude, que la formulation et le style de l'étudiant ne copient pas servilement ceux de l'auteur.

g) Révision de la contraction

C'est l'étape finale. Celle où l'étudiant vérific la correction de la langue (orthographe, accords, constructions, propriété des termes, style) et la présentation de son travail (vérification du nombre de mots).

4 C'est ce que démontre l'expérience de Denhiere rapporté par Sprenger-Charolles (1980). 


\section{Application}

Nous allons maintenant présenter un exemple d'application des principes exposés ci-dessus. I.e texte choisi offre une difficulté particulière dans la mesure oì il s'agit d'une interview avec plusicurs personnes. Nous avons conservé le document sous sa forme authentique (avec les éléments d'information sur les intervenants), afin de permettre à l'étudiant de mieux comprendre les conditions de production et la situation d'énonciation.

\subsection{Consigne}

Voici un exemple de consigne:

Après avoir lu le texte suivant (1240 mots), vous en rédigerez une contraction au $1 / 4$, soit 310 mots, avec une tolérance de $10 \mathrm{p}$. cent, en plus ou en moins. Vous donnerez un titre (de type informatif) à votre contraction.

\subsection{Texte à contracter}

Un entretien avec Michel Callon et Jean-Pierre Lévy-Leblond:

\section{La science et l'écrit: la machine folle}

La science moderne n'aurait jamais pu exister sans l'invention de l'imprimerie. Mais aujourd hui, on croule sous des milliers d'articles savants fue persomue ne lit.

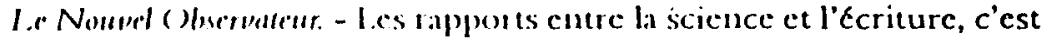
un vaste sujet. Commençons par constater que les scientifiques écrivent beaucoup...

Michel Callon. - Les scientifiques et les technologues sont avant tout des écrivains. Une très grande part de leur activité - sinon la plus grande - consiste à rédiger des articles pour rendre compte de leurs travaux dans les revues spécialisées. Et à lire ceux des autres, pour les utiliser, critiquer, réfuter, s'en servir comme point de départ pour écrire eux-mêmes d'autres papiers, et ainsi de suite. Dans leur très grande majorité, les mots nouveaux sont créés par les chercheurs, et la communauté scientifique est une population d'obsédés d'écriture...

Jean-Marc Léry-Leblond. - Au point que l'on croule sous l'hyper-inflation de ces articles savants. Le système de la publication scientifique a très bien 
fonctionné pendant un siècle et demi, disons tant que la recherche est restée une activité artisanale et que chacun pouvait lire tout ce qui le concernait. Mais depuis les années 50, ce système s'est emballé. Aujourd' hui, il est à la veille de son effondrement et, en tout cas, il ne remplit plus sa fonction initiale, qui était la circulation de l'information. Il y a trop de revues, toujours plus spécialisées, lues par de moins en moins d'abonnés et publiant des articles de moins en moins originaux et intéressants. De toute façon, les résultats scientifiques circulent d'abord par le téléphone, par le fax, par le courrier électronique, ou par prétirages avant parution. La publication scientifique traditionnelle ne sert pratiquement plus à rien.

N.O. - Comment en est-on arrivé là?

J.-M. Lévy-Leblond. - Deux grandes raisons: le système d'évaluation des scientifiques a pris comme critère quasi unique la fréquence des publications, ce qui incite à publier sans retenue. Et les éditeurs spécialisés ont poussé à la roue, multipliant les revues dans des domaines de plus en plus pointus, sachant qu'elles avaient une clientèle obligée, un marché captif. Résultats: une masse d'informations publiées inutilement. Une preuve: $60 \%$ à $80 \%$ des articles publiés ne sont jamais cités, ou alors seulement par leurs auteurs dans leurs propres articles subséquents.

M. Callon. - D'après les statistiques dont je dispose, ce sont $80 \%$ des articles parus qui ne sont jamais cités par un autre auteur. Et les citations, lorsqu'elles existent, ont des significations variables qui n'excluent pas le copinage pur et simple. Il faut bien comprendre ce qu'est un article scientifique dans une revue spécialisée. Cette sorte de littérature a trois caractéristiques: 1) Elle est truffée de chiffres, diagrammes, schémas et tableaux qui apportent la preuve de ce qui est écrit; 2) Elle utilise un vocabulaire extraordinairement codifié et particulier, chaque fraction d' une sous-discipline constituant une tribu au langage incompréhensible pour les autres tribus-on peut reconnaître la tribu à son vocabulaire même si on ne le comprend pas; 3) Chaque article est assorti de quelques dizaines de citations on references renveyant a d'antres articles. Du coup la mesure des citations est en eflet ues instructive. On a pu établir qu un chercheur nobélisable a été cité en moyenne 4000 on 5000 fois...

N.O. - Il y a donc des gens qui passent leur temps à éplucher tout ça?

M. Callon. - Bien sûr. L'analyse des publications scientifiques est devenue une discipline scientifique à part entière - la scientométric - qui publie ses propres articles... Aux Etats-Unis, il existe un institut spécialisé. On y emploie 300 personnes qui lisent les 3500 revues les plus importantes uniquement 
pour explorer les citations qui suivent chaque article. La mise en évidence de communautés de chercheurs qui se citent mutuellement et les analyses de vocabulaire fournissent des renseignements très précieux et donnent une véritable cartographie de la science. On distingue tout de suite les domaines les plus actifs et les plus dynamiques. Heureusement tout ce travail bénéficie d'outils informatiques de plus en plus puissants.

N.O. - Linformatique, parlons-en. Les nouveaux outils de communication électronique n'offrent-ils pas une solution à ce problème de l'inflation des publications?

J.-M. Lếry-Leblond. - Oui et non. Bien sûr, si, au lieu d'être imprimés dans des revues, les articles scientifiques étaient diffusés sur écran par télématique, chacun étant libre de ne sortir sur son imprimante que ceux qui l'intéressent, déjà on désencombrerait les rayons des bibliothèques et on économiserait des tonnes de papier. Mais l'une des fonctions de base de la publication ne serait plus remplie: dans les revues, avant publication, chaque article est vérifié par un comité de lecture. Des referees doivent donner le feu vert. Avec la télématique, si chacun devenait libre de déposer directement, sans aucun contrôle, son petit travail dans une banque de données, ce serait bien pis: il $\mathbf{n}$ y aurait plus aucun filtre, l'inflation en serait encore accélérée.

Resterait de toute façon un problème de fond: en recherchant un article sur écran, à l'aide de mots clés ou de programmes d'indexation automatique, on ne trouve que ce que l'on connaît déjà. On se prive des avantages du hasard. Ce qui féconde véritablement une recherche, c'est de feuilleter une revue et de tomber sur un article inattendu, dans un domaine peut-être très différent, qui apporte une idée nouvelle que l'on n'aurait jamais pensé demander par un mot clé. On ne sortira pas de la crise en se privant du papiequ aucun écran au monde ne remplacera jamais-mais en imaginant un moyen pour réduire le nombre des articles inutiles.

Remarquons en passant que si la publication scientifique spécialisée est en rrise, il n en va pas de même de l'édition des ouvrages scientifipues destinés au grand public ou an public cultive. En France en particulier, l'édition scientilique se porte très bien. Les tirages augmentent et la qualité des livres aussi. Une des raisons: les chercheurs ont compris qu'il était de leur devoir de s'adresser-aussi-au grand public. Autrefois, c'était plutôt mal vu dans le milieu, mais les mentalités ont changé.

N.O. - La science aurait-elle pu naître sans l'imprimerie?

J.-M. Lévy-Leblond. - La science moderne n'aurait jamais pu exister sans l'invention de l'imprimerie, pas plus que la science antique n'aurait pu 
apparaître sans l'invention de l'écriture.

M. Callon. - De même que l'alphabet a permis des opérations mentales impensables sans lui, l'imprimerie a renouvelé les conditions mêmes de la création scientifique. Il $\mathrm{n}$ y aurait jamais cu de révolution copernicienne si Copernic n'avait pu réunir et comparer, sur sa table de travail, tous les ouvrages de cosmologie ecrits avam lui. Non seulement cela aurait éte tres difticile a réliser avec des manuscrits. Mais de plus les manuscrits etaient trulles d'erreurs. Avec l'imprimerie, avant de se lancer dans un tirage en grande série, on se sent forcément obligé de vérifier, corriger, critiquer.

N.O. - Pour la science, l'imprimerie est définitivement irremplaçable?

J.-M. Lévy-Leblond. - Le papier imprimé a un très bel avenir devant lui si on sait ne pas en abuser...

\section{Propos recueillis par Fabien Gruhier}

Michel Callon est directeur du Centre de sociologie de l'innovation à l'École des mines et codiręteur avec Bruno Latour de la collection Anthropologie des sciences et des techniques aux éditions de la Découverte.

Jean-Marc Lévy-Leblond est physicien, professeur à l'université de Nice, directeur de la collection Science ouverte aux éditions du Seuil et de la revue Alliage.

(Le Nouvel Observateur, 17-23 octobre 1991)

\subsection{Exploilation}

Les exercices qui suivent sont fondés sur l'exploitation des principes présentés dans la section 1. Leur objectif est de mener graducllement l'étudiant vers la rédaction de la contraction proprement dite. Leur difficulté pourra varier selon le type de clientèle, en particulier selon le niveau des élèves ou des étudiants (secondaire, collégial, universitaire) ou selon qu ils travaillent en langue maternelle ou cn langue seconde ou étrangère.

\subsubsection{Compréhension du texte}

On a souvent comparé la contraction de texte à la traduction. Cet exercice serait une sorte de version du français au français. Malgré l'approximation d'une telle comparaison, il partage avec la traduction la nécessité de bien comprendre le texte de départ pour éviter de faire des faux-sens ou des contresens. Les questions qui suivent favoriseront cette compréhension:

\section{- Situation d'énonciation}

1) Qui est le producteur du texte? 
2) Qui est l'émetteur du message?

3) Qui en est le destinataire?

- Typologic textuclle

1) De quel type de cexte s'appit-il?

5) (Quelle est la linme dutexte?

- Le message

6) Quel est le sujet du texte?

7) Quel en est l'angle?

8) Quel en est le message essentiel?

- Questions particulières au texte choisi

9) Quelles sont les principales activités des scientifiques?

- Compréhersion du texte et des allusions culturelles

Il convient, dans ce cas précis, d'expliquer rapidement aux étudiants la place et le rôle des publications dans l'activité des chercheurs et dans la recherche scientifique. De plus, quelques questions particulières permettront de vérifier si les étudiants ont bien compris et s'ils ne risquent pas de faire des erreurs d'interprétation. Par exemple:

1) Dites à quelle époque (approximativement) et dans quelle région du monde a été inventé l'alphabet?

2) En quelle année a été inventée l'imprimerie?

3) De quand date la télématique?

4) Qui était Copernic?

5) Quelle découverte l'a rendu célèbre?

- Compréhension du vocabulaire

Il convient de faire vérifier dans un dictionnaire le sens des mots qui peuvent faire difficulté. Par exemple: technologue, courrier électronique, prétirage, critère, problème de fond, cosmologie, referee, etc.

\section{- Figures de rhétorique}

La compréhension du rôle et de la signification des figures de rhétorique est essentielle non seulement à la compréhension du texte, mais aussi à la préparation de la contraction. Dans notre exemple (ce n'est pas forcément le 
cas pour d'autres textes), il est utile d'attirer l'attention de l'étudiant sur les métaphores.

1) Qu est-ce qu une métaphore (explication rapide; exemple)?

2) Relevez les métaphores du texte.

- Registres et niveaux de langue

Comme nous avons choisi d'exiger un style neutre dans la contraction, ${ }^{5}$ il est important de sensibiliser les étudiants aux éventuelles différences de niveaux de langue dans le texte donne.

1) Quels sont les niveaux de langue (explication rapide) le plus souvent indiques par le dicrionnaire que vous utilise $z$ ?

2) Relevez les mots didactiques du texte.

3) Relevez les motsfamiliers.

\subsubsection{Etablissement du plan de la contraction}

a) Extraction des idées

Lobjectif de la contraction, comme le terme même le suggère, consistant à réduire le texte de départ, il faut opérer un choix parmi les idées à retenir, en partant du principe que certaines sont plus importantes que d'autres. $\mathrm{Ce}$ choix doit se faire à la lumière du message essentiel. Le nombre d'idées retenues dépend de la proportion de la contraction: plus la proportion est élevée, plus le tri sera sévère. A cette étape, nous conseillons simplement de souligner dans le texte les idées à retenir. Trois sortes d'idées sont à éliminer:

- Les idées secondaires

Les idées secondaires sont celles dont la présence n'est pas nécessaire à la compréhension du message essentiel.

- Les idées redondantes

Celles qui apparaissent plus d'une fois dans le texte on dans la phrase.

Dans notre exemple, nous observons plusieurs cas de redondances au niveau de l'ensemble du texte. Par exemple: il y a trop de revues, toujours plus spécialisées et multipliant les revues dans des domaines de plus en plus

5 C'cst le contenu, le message qui nous intéresse. De plus, cela permet mieux d'éviter le plagiat. 
spécialisés. Au niveau de la phrase, remarquons le savoureux pléonasme: le papier imprimé a un très bel avenir devant lui ...

Exercice: Relevez les idées et les expressions redondantes.

\section{- Les digressions}

Ce sont les idées qui s'écartent du sujet. Dans notre exemple, nous pouvons considérer l'allusion à la création des mots (Dans leur très grande majorité, les mots nouveaux sont créés par les chercheurs) comme une idée faisant digression.

Exercice: Relevez d'autres idées qui sont en fait des digressions.

\section{b) Nominalisation des idées retenues}

La nominalisation consiste à reformuler en phrases nominales les idées retenues. Cette transformation offre plusieurs avantages. C'est une étape utile avant la réduction nécessaire des éléments trop développés. Elle permet d'éliminer la gangue et de dégager l'idée dans toute sa pureté. Elle sert à se démarquer du style de l'auteur. Enfin, elle est un excellent exercice de gymnastique intellectuelle et grammaticale. Plusieurs cas de figure sont possibles:

- Identité entre le signe de départ et le signe d'arrivée, l'idée reste la même, seule la catégorie grammaticale change. Par exemple:

Les mots nouveaux sont créés par les chercheurs: Création par les chercheurs des mots nouveaux (Idée de créer sous formes verbale et nominale).

- Différence entre le signe de départ et le signe darrivée:

- Passage, pour exprimer la même idée, d'un verbe à un nom. Par exemple:

Lanalyse des publications est devenue une discipline scientifique: Promotion de l'analyse des publications an rang de discipline scientilicue.

- Passage d'uin adverbe à an nom. Par cxemple:

Il y a trop de revues (. . .) lues parde moins en moins d abonnés: Diminution du nombre d abonnés.

Exercice: Nominalisez les idées retenues.

c) Réduction

Une première élimination au niveau des idées ayant été faite, il reste à 
poursuivre le travail de réduction au sein même des idées retenues. Ces réductions se situent au niveau de la proposition ou du syntagme.

- Réduction de termes coordontiés

- Réduction par le choix d'un des deux termes du texte (réduction interne: le plus particulier s'efface au profit du plus général). Fxemple: les scientifiques pour les scientifiques et les teclmologues.

- Réduction par le choix d'un terme absent du texte ou de la phrase (réduction externe: les deux termes considérés comme insuffisamment généraux s'effacent au profit d'un hypéronyme). Exemple: les chercheurs pour les scientifiques et les technologues.

- Réduction par le choix d'un terme exprimant la fin du processus, le plus haut degré d'une qualité, etc. Exemple: réınir el comparer: comparer.

Exercices:

- Relevez les séries (para)synonymiques. Exemple: Scientifiques, technologues, chercheurs, auteurs, communauté scientifique, communauté de chercheurs, population d'obsédés de l'écriture, tribu.

- Relevez dans une série donnée le mot le plus général (hypéronyme). Exemple: Article, papier, article savant, publication, ouvrage scientifique, livre, prétirage (dans ce cas: publication).

- Trouvez en dehors d'une série donnée un mot plus général. Exemple: Chiffres, diagrammes, tableaux, schémas (dans ce cas: illustrations).

- Précisez les différences d'emploi des termes d'une série (para)synonymique.

- Réduction de termes juxtaposés

- Réduction par hypéronyme. Excmple: par le telephome, par le fax, par le courrier électronique: par la télématique.

- Suppression, reclassenent et coordination. Exemple: pour les utiliser, critiquer, réfuter, s'en servir comme point de départ: pour les utiliser et les critiquer.

Exercice: Relevez des séries de termes juxtaposés et réduisez-les à un ou à deux termes.

- Réduction d'une série de syntagmes discontinus

Une même idée peut être exprimée plusieurs fois, à des endroits et avec des moyens différents. Il convient de repérer ce genre de procédé et d'opérer les réductions qui s'imposent pour éviter toute redondance dans la contraction du texte. 
Voici un exemple:

Les scientifiques et (.. ) sont avant tout des écrivains. Une très grande part de leur activite-sinon la plas grande _-consiste à rédiger (. . .): Prééminence de la rédaction daus l'activité des clicrcheurs.

Exercice: Soulignez les syntagnes qui expriment une même idéc et réduisez-les à une formulation mique.

- Réduction des expansions

Une expansion est un élément de la phrase (mot, syntagme, proposition) qui vient ajouter un élément d'information utile, mais non indispensable, à la compréhension du message essentiel.

- Fusion de deux indépendantes. Exemple: Chaque article est vérifié par un comité de lecture. Des referees doivent donner le feu vert: Chaque article doit être accepté par un comité de lecture .

- Réduction de la principale plutôt que la subordonnée. Exemple: les éditeurs spécialisés ont poussé à la roue, multipliant les revues dans des domaines de plus en plus pointus: les éditeurs ont multiplié les revues. L'élément stylistique est sacrifié au profir de l'élément informatif.

- Effacement d'expansions non essentielles. Exemple: en recherchant un article à l'écran, à l'aide de mots clés ou de programmes d'indexation automatique, on .... en recherchant un article par télématique. La connaissance que le lecteur partage avec le rédacteur permet au premier de comprendre le second sans que plus de précisions soient nécessaires.

\section{d) Etude des connecteurs}

Un texte est un ensemble hiçrarchisé d'idées, Les relations, les passages, d'une iclece a une antte se font soit par simple progression logique, sans marqueurs particuliers, lorsque le sens des idées suffit pour permettre la transition (ce sont les mots portcurs de sens qui participent à la progression du texte), soit au moyen de mots spéciaux, les connecteurs, qui peuvent être des adverbes, des locutions, des conjonctions de coordination ou de subordination, etc.

Il est très important de bien repérer ces mots dans le texte et d'en dégager le sens. Ces marqueurs peuvent faciliter la transition entre deux mots, deux propositions, deux phrases ou deux paragraphes.

Exemples de relations logiques marquées par des connecteurs:

- Paragraphe 1

1. Prééminence de la rédaction dans l'activité des scientifiques et 
2. Importance de la lecture dans l'activité des scientifiques

$=$ addition

- Paragraphe 2

2.1. Efficacité du système traditionnel de publication scientifique au stade artisanal de la science mais

2.2. Inaptitude du système à remplir sa fonction depuis les années 50

$=$ opposition

- Paragraphes 1 et 2

1. Préminence de la rédaction dans l'activité des scientifiques au point que

2. Surabondance des articles savants

= conséquence

Exercices: Dans le paragraphe 1 et dans le paragraphe 3, inscrivez dans un rectangle les mots qui Eavorisent la progression des idées. Dans le paragraphe 2, inscrivez dans un cercle les connecteurs (adverbes, conjonctions, locutions) qui favorisent la progression des idées.

\section{e) Hiérarchisation des idées}

En distinguant les idées générales et les idées secondaires, nous avons déjà fait des distinctions hiérarchiques entre les différentes idées du texte. En effet, toutes ne sont pas aussi importantes. En dégageant le message essentiel, nous avions en fait déjà défini l'idée principale du texte. Puis nous avons dégagé l'idée principale (ou les idées principales) et les idées secondaires de chaque paragraphe.

Exemple de hiérarchisation: Dans l'exemple qui suit, nous voulons montrer par l'usage de la numérotation décimale l'organisation hiérarchique du paragraphe (Il s'agit du par. 2).

2. Imminence de l'effondrement du système traditionnel de publication scientifique;

2.1. Efficacité du systène traditionnel de publication scicntifinue au stadeartisanal de la science;

2.2. Inaptitude actuelle du système traditionnel de publication scientifique à faire circuler l'information;

2.2.1. Surabondance de revues de plus en plus spécialisées;

2.2.2. Diminution du nombre des abonnés;

2.2.3. Diminution de l'intérêt des articles; 
2.3. Apparition d'autres canaux de circulation de l'information scientifique.

Exercice: Sur le modèle qui précède, présentez une hiérarchisation du paragraphe 4.

\subsubsection{Rédaction de la contraction}

Il est encore possible à cette étape de travailler la concision, cette fois au niveau de la formulation. Voici quelques procédés utiles:

- Substitution d'un mox simple a un mot compose on a une locution. l'ar excmple: pousser a la roue: aider; rendre compte de: présenter.

- Substitution de la forme affirmative à la forme négative.

- Substitution de la voix active à la voix passive.

- Substitution d'une épithète à une relative.

Exercice: Relevez les mots composés et remplacez-les par des mots simples.

\section{Corriges}

\subsection{Plan de la contraction}

1. Prééminence de la rédaction dans l'activité des scientifiques; Egalement:

1.2. Importance de la lecture;

(1.3. Création de la majorité des mots nouveaux par les chercheurs.) Au point que:

2. Imminence de l'effondrement du système de publication scientifique;

2.1. Efficacité du système au stade artisanal de la recherche;

2.2. Inaptitude actuelle du systeme à faire circuler l'information A cause de:

2.2.1 Surabondance des revues;

2.2.2. Diminution du nombre des abonnés;

2.2.3. Diminution de l'intérêt des articles.

Origines de la cause:

3.1. Prééminence du critère quantitatif dans l'évaluation des scientifiques; 
3.2. Multiplication des revues hyperspécialisées;

Avec comme conséquence:

3.3. Pourcentage élevé d articles jamais cités par d'autres scientifiques $(80 \%)$.

I:Il cflet:

4. Caractéristiques d'un article scientifique:

4.1. Illustration de la preuve (chiffres, tableaux et schémas);

4.2. Spécificité du langage;

4.3. Renvois à d'autres articles (citations, références).

D'oì:

5.1. Promotion de lanalyse des publications au rang de science (scientométrie);

5.2. Existence d'un copinage entre chercheurs qui se citent mutuellement;

6.1. Conséquences positives de la diffusion par télématique sur les bibliothèques et l'utilisation de papier;

Annihilées cependant par:

6.2. Suppression du rôle de contrôle des comités de lecture;

\section{Egalement:}

7.1. Suppression de l'avantage du hasard des lectures pour la naissance d'idées nouvelles;

Donc:

7.2. Nécessité non pas de se passer du papier, mais de trouver le moyen de réduire le nombre des publications.

Par contrasie:

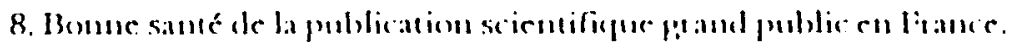

En fait:

9. Dépendance de la science modernc par rapport à l'imprimerie.

Grâce d̀ elle:

10.1. Possibilité de comparer plusieurs ouvrages;

10.2. Plus grande fiabilité des textes;

10.3. Exemple de Copernic.

En conclusion:

11. Confiance en l'avenir de l'imprimé dans le domaine de la publication scientifique. 


\subsection{Contr: de texte}

\section{- du système de publication scientifique}

Le consacrent la majeure partic de leur activité a lire ct a rédiger : ...nut que la science est restéc à un stade artisanal, le système de publication scientifique a rempli sa fonction de diffusion de l'information. Mais aujourd hui, il risque de s'effondrer à cause de la surabondance d'articles de moins en moins intéressants et de revues de plus en plus spécialisces.

La cause de cette inflation réside dans le fait que la fréquence de publication est devenue le principal critère d'évaluation des chercheurs et que les éditeurs ont poussé à la création de revues hyperspécialisées. Si bien qu on constate, aujourd hui, que $80 \%$ des articles ne sont jamais cités par d'autres que leurs auteurs...

Il faut bien comprendre qu'un article scientifique se distingue par trois éléments: des chiffres et des illustrations pour étayer la preuve, un langage compréhensible aux seuls initiés et des citations d'autres articles. L'analyse de ces citations est devenue une véritable science, la scientométrie. Une science qui révèle, entre autres, l'existence d'un copinage entre chercheurs qui se citent mutuellement...

Si les scientifiques décidaient de diffuser leurs travaux par la télématique, cela permettrait certes de désencombrer les bibliothèques et d'économiser du papier. Mais la disparition des comités de lecture, qui jouent un rôle de contrôle, aurait pour conséquence d'augmenter encore le nombre des publications. I) phus, la recherde telématique par mots clés écarte la possibilité

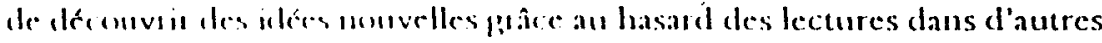
domaines.

Il convient donc, non pas de se passer de l'imprimé, mais plutôt de réduire le nombre des publications. D'ailleurs la science moderne s'est constituée grâce à l'imprimerie, qui a permis de réunir et de comparer un grand nombre d'ouvrages sur un même sujet, et produit des textes beaucoup plus frables que les manuscrits.

Il est donc certain que l'imprimé a encore un bel avenir dans le système des publications scientifiques.

Nombre de mots : 334 . 


\section{Références}

Manuels pratique de contraction de texte

Arambourou, Ch., Texier, F., \& Vanoye, F. (1991). Guide du résumé de texte. Paris: Hachette.

Baron-Dejours, C., \& Drifter, M. S. (1991). Français. Etapes B.E.P. Paris: Nathan.

Bernard, J. (1985). Méshodologie pratique du résumé de texte et de la synthèse de documents. Paris: Hatier.

Brillant, A., \& Dejean, J. L. (1991). Corrigés de fraņ̧ais. Paris: Hatier.

Carloni, J. C. (1984). Pratique de la contraction de texte aux concours des grandes écoles. Montreuil: Bréal.

Clerc, G. (1992). 50 modèles de résumés de textes. Alleur: Marabout.

Dambre, M. (1985). Du résumé à la discussion. L'épreuve anticipée au bac. Paris: Nathan.

Finniss, F. (1985). Contraction de textes. Paris: Vuibert.

Gaillard, P., \& Launay, C. (1982). Le résumé de texte. Paris: Hatier.

Geray, Ch. (1983). Bonnes copies du bac. Franģais. contraction de texte, résumé ou analyse. Paris: Hachette.

Geray, Ch., \& Desalmand, P. (1989). Franģais. Technique du résumé et de la discussion. Paris: Hatier.

Gourmelin, M. J. (1990). Le résumé de texte. Paris: La Documentation française.

Jeoffroy-Faggianclli, R., \& Plazolles, I. R. (1975). 'lidmigues de lexpersion a de la communication. Paris: Nathan.

L.ane, Ph., \& Rive, J. (1989). Itangais: Le resume de rexte. Paris: Hachete.

Moreau, J. (1977). La contraction et la synthèse de textes. Paris: Nathan.

Morfaux, L. M., \& Prevost, R. (1990). Résumé et synthèse de textes. Paris: A. Colin. 
Pouzalgues-Damon, E. (1991). Frangais. Contraction de texte, Questions de compréhension, Commentaires. Paris: Nathan.

Robrieux, J. J. (1988). Le français au BTS. Résumé. Paris: Dunod.

Rohou, J. (1990). Les devoirs de français. Rennes: Presses universitaires de Rennes.

Russell, P. (1988). How to 1urite a précis. Ottawa: University of Ottawa Press.

Stalloni, Y. (1990). Le résumé au bac. Paris: Bordas.

Stalloni, Y. (1986). Méthode de contraction et de synthèse de textes. Paris, éd: Ellipses-Marketing.

Tort, P., \& Desalmand, P. (1986). Méthodologie pratique du résumé de texte. Paris: Hatier.

\section{Études théoriques}

Charolles, M. (1991). Le résumé de texte scolaire. Fonctions et principes d'élaboration. Pratiques, 72, 7-32.

Clement, L. (1985). La contraction de texte: problèmes théoriques et . pratiques. Revue internationale de philosophie, 155, 413-433.

Groupe de recherches sur la condensation de l'information (1980-1983).

Condenser. 4 numéros, Saint-Etienne: Faculté des lettres et sciences humaines, Institut de linguistique.

Dumont, D., \& Audet. Ch. H. (1986). La contraction de texte. Québec franşais, 57-64.

Gautherot, J. M. (1972). La contraction de texte. Le franģais dans le monde, $89,29-3.5$.

Hadjadj, 1). (1982). I a contraction de texte: Bilan et perspectives. I.e frangais anjourd'hui, 60, 21-33.

Jomand-Baudry, R. (1991). Le résumé de texte au baccalauréat. Pratiques, $72,105-124$.

Laurent, J. P. (1985). L'apprentissage de l'acte de résumer. Pratiques, 48, 7189. 
Maldidier, D., \& Normand, Cl. (1982). Passer d'un discours à un autre: la contraction de textc. Langue française, 53, 109-122.

Petroff, A. (1975). Méthodologie de la contraction de textc. Langue française, 26, 41-55.

Schnedecker, C. (1991). Resumer: Gammes d activite. Pratiques, 72, 55-90.

Sprenger-Charolles, L. (1980). Le résumé de texte. Pratiques, 26, 59-90.

Van Dijk, T. A. (1977). Semantic macro-structures and knowledge frames in discourse comprehension. M. A. Just \& P. A. Carpenter (eds): Cognitive processes in comprehension, Hillsdale, N. J. : Erlbaum, 3-32. Trad. française dans: Denhiere, G. (1984): Il était une fois, Lille, Presses de l'université de Lille, 49-84.

Van Dijk, T. A. (1977). Text and context. Exploration in the semantics and pragmatics of discourse. London \& New York: Longman.

Veck, B. (1991). Réduire/Traduire : L'épreuve du résumé. Pratiques, 72, 91104.

Vigner, G. (1991). Réduction de l'information et généralisation: Aspects cognitifs et linguistiques de l'activité de résumé. Pratiques, 72, 33-54. 